\title{
The gifts that keep on giving: physiological functions and evolutionary dynamics of male seminal proteins in Drosophila
}

\author{
MF Wolfner \\ Department of Molecular Biology and Genetics, Cornell University, Ithaca NY 14853-2703, USA
}

\begin{abstract}
During mating, males transfer seminal proteins and peptides, along with sperm, to their mates. In Drosophila melanogaster, seminal proteins made in the male's accessory gland stimulate females' egg production and ovulation, reduce their receptivity to mating, mediate sperm storage, cause part of the survival cost of mating to females, and may protect reproductive tracts or gametes from microbial attack. The physiological functions of these proteins indicate that males provide their mates with molecules that initiate important reproductive responses in females. A new comprehensive EST screen, in conjunction with earlier screens, has identified $\sim 90 \%$ of the predicted secreted accessory gland proteins (Acps). Most Acps are novel proteins and many appear to be secreted peptides or prohormones. Acps also include modification enzymes such as proteases and
\end{abstract}

their inhibitors, and lipases. An apparent prohormonal Acp, ovulin (Acp26Aa) stimulates ovulation in mated Drosophila females. Another male-derived protein, the large glycoprotein Acp36DE, is needed for sperm storage in the mated female and through this action can also affect sperm precedence, indirectly. A third seminal protein, the protease inhibitor Acp62F, is a candidate for contributing to the survival cost of mating, given its toxicity in ectopic expression assays. That male-derived molecules manipulate females in these ways can result in a molecular conflict between the sexes that can drive the rapid evolution of Acps. Supporting this hypothesis, an unusually high fraction of Acps show signs consistent with their being targets of positive Darwinian selection.

Heredity (2002) 88, 85-93. DOI: 10.1038/sj/hdy/6800017

Keywords: accessory gland; reproduction; protease inhibitor; ovulation; peptide hormone; rapid evolution

\section{Introduction}

The recognition between members or cells of the opposite sex is an important ingredient in the success of a mating. The sexes can also influence each other after mating. The focus of this article is on protein 'gifts' that a male Drosophila gives his mate in the seminal fluid. The proteins on which this article centers are the products of genes expressed only or primarily in the accessory glands of male flies. These genes are downstream targets of the regulatory hierarchies that determine sexual phenotype (see Zarkower (2001) for review of such hierarchies). Once transferred to a female during mating, seminal proteins influence the female's reproduction, behavior and physiology. This article will first review briefly what these seminal proteins are and how they were found, and their general functions in females. Then the focus will be on the function of two seminal fluid proteins, one that regulates ovulation and one that regulates proteolysis and is proposed to affect the mated female's lifespan. Finally, results that point to interesting evolutionary dynamics of seminal proteins will be described. These proteins provide the gift of molecular tools with which to address some hypotheses of evolutionary interest.

Correspondence: MF Wolfner, Department of Molecular Biology and Genetics, Cornell University, Ithaca NY 14853-2703, USA. E-mail: mfw5@cornell.edu
What protein gifts does a male fly give his mate, and what do they do to her?

During courtship in Drosophila, males use chemical and visual cues to find females. Once a male has detected a female, he orients towards her, extends a wing, and vibrates it to produce a species-characteristic mating song (reviewed in Hall, 1994; Greenspan and Ferveur, 2000). A female who is receptive to mating (sexually mature and unmated or not recently mated), and who recognizes the song as from her own species, will modify her behavior, permitting the male to reach her and continue the courtship ritual. Ultimately the male will mount the female and copulate with her. After mating, several aspects of the female fly's physiology and reproductive behavior are profoundly changed (see Chen, 1996; Kubli, 1996; Wolfner, 1997 for reviews of, and references to, earlier work). First, whereas an unmated female Drosophila melanogaster produces and lays a couple of (unfertilized) eggs a day, after mating a female's egg-laying levels increase by an order of magnitude (with consequent increases in oogenic and ovulation rates). Second, before mating a female is receptive to mating with a male from her species, but after mating she will actively reject males by kicking them away and by extruding her ovipositor. This effect on the female's receptivity can be considered to be the result of a chemical version of the mate-guarding seen in several insects (see Krebs and Davies, 1993; Alcock 1998 for review of mate-guarding). Third, mated 
females also differ from unmated females by storing sperm. Fourth, a mated female has a shorter lifespan than an unmated female (Fowler and Partridge, 1989). Almost all these changes in their full magnitude require that the female mated: it is not enough for her only to see, hear, or smell the male, no matter how enticing, graceful or charming one might think he could be. The differences between mated and unmated females indicate that copulation per se is required to elicit the changes in the female's reproductive behavior and physiology. Additional experiments showed that the full spectrum and magnitude of the changes requires that the female have received seminal fluid and sperm from her mate (Manning, 1962; Hihara, 1981; Kalb et al, 1993; Harshman and Prout, 1994; Chapman et al, 1995; Tram and Wolfner, 1998, 1999; Xue and Noll, 2000; Heifetz et al, 2001). [Males have also been reported to donate cuticular hydrocarbons, and elemental phosphorus, to their mates (for reviews, see Antony and Jallon, 1982; Jallon, 1984; also see Markow et al, 2001). Consideration of these, and of the contributions of the male's ejaculatory duct and bulb (eg Gilbert et al, 1981; Cavener and MacIntyre, 1983; Ludwig et al, 1991; Lung et al, 2001b) is beyond the scope of this review.]

Sperm and seminal fluid proteins both exert postmating influences on female insects. In D. melanogaster, genetic techniques can be used to tease apart their relative contributions. Flies lacking sperm can be generated because of mutations that prevent germ cell formation (eg Boswell and Mahowald, 1985); flies of the X0 chromosome constitution also lack functional sperm (Bridges, 1916). These spermless flies still make and transfer seminal proteins. Comparisons of the phenotypes of mates of spermless flies to mates of flies that make sperm have identified several general reproductive roles of sperm. First, and obviously, sperm are needed to fertilize eggs. Second, the presence of sperm in females contributes to some of the initial post-mating changes, such as increased oogenic and egg-laying rates (Xue and Noll, 2000; Heifetz et al, 2001). Stored sperm are also needed to maintain the mated state: long-term inhibition of mating receptivity, and continued elevated rates of oogenesis, ovulation and egg deposition all require the presence of sperm in the female (Manning, 1962, 1967; Kalb et al, 1993; Tram and Wolfner, 1998; Xue and Noll, 2000; Heifetz et al, 2001). Finally, sperm from different mates compete within a multiply-mated female for their use in fertilizing eggs (Parker, 1970). This competition between gametes from different males is likely to be of evolutionary importance.

Analogous experiments identified general roles of Drosophila seminal proteins, particularly those produced in the accessory gland of the male fly (these proteins are called 'Acps' for accessory gland proteins). Flies that fail to make proteins in the main cells of their accessory glands can be generated by forcing those cells to produce an intracellular toxic protein; these flies lack seminal proteins but, for technical reasons unrelated to seminal proteins, also lack sperm (Kalb et al, 1993). Recently, a fly line was reported to produce sperm in the absence of development of accessory glands (Xue and Noll, 2000). This line is mutated in the paired gene, whose functions include specifying accessory gland development (Xue and Noll, 2000). Results from the two types of fly strains just mentioned have identified roles for Acps in the post-mating changes in female flies. Short-term action of Acps is needed to increase egg production/laying process (including increased rates of oogenesis, ovulation and egg deposition) and decrease receptivity to re-mating. Changes induced by Acps last for up to a day post-mating; continuation of the changes beyond this time requires the presence of stored sperm in the female. Rapid-acting, but temporary, Acps could be advantageous to both male and female. It takes at least $1 \mathrm{~h}$ to store sperm to maximal levels (Gilbert, 1981; Tram and Wolfner, 1999) and potentially longer for the presence of stored sperm to be manifested in behavioral or physiological changes in the female. Seminal proteins quickly change the female's physiology/behavior after mating, even while sperm are being stored. However, it would seem disadvantageous for females to produce high numbers of eggs and to avoid mating if they did not receive sperm from the mating, or after their stored sperm have been depleted. From this perspective, it seems advantageous to tie long-term persistence of these changes to the presence of stored sperm in the female. Seminal proteins are also necessary for the efficient storage of sperm by females (Tram and Wolfner, 1999). At least in part as a consequence of this (Chapman et al, 2000), they are likely to play roles in sperm competition. Indeed, a role for Acps in sperm competition has been reported (Harshman and Prout, 1994), and a correlation has been observed between alleles at four Acp loci and levels of sperm displacement in lines carrying chromosomes isolated from the wild (Clark et al, 1995). Seminal proteins also decrease the lifespan of the mated female (Chapman et al, 1995). Acps have roles in addition to those determined by the genetic studies: Acps include proteins with antibacterial activity (Lung et al, 2001a), suggesting a role in protecting the reproductive tracts (of either sex), or sperm, or the first egg laid after mating, from microbial attack. In addition, at least one Acp is a component of the gelatinous mating plug that forms in the reproductive tract of the mated female (Lung et al, 2001b). This structure is thought to assist in the movement of sperm into the female and into storage (Bairati, 1968).

In sum, the male provides his mate with the gifts of sperm and seminal proteins, which have profound effects on female reproductive physiology and behavior. It should be made clear that females are by no means passive players in the reproductive exchange between the sexes (Eberhard, 1996). There is evidence, for example, that $D$. melanogaster females play a role in sperm competition (Clark and Begun, 1998; Clark et al, 1999). Nevertheless, most molecular studies of effectors of post-mating changes in Drosophila females have focused on malederived proteins. Technical considerations largely explain this apparent bias. It is easier to identify the products of a single male tissue, the accessory gland, than it is to isolate effectors of reproductive behavior/physiology among the greater complexity of all the female's tissues. In addition, it is easier to identify factors that cause a change beginning at a defined time-point of introduction (in this case, introduction from the male during mating) as compared with the effects of regulatory molecules that are continually present in female flies.

\section{How are Drosophila seminal fluid proteins identified, what are their features and how many are there?}

The rest of this article focuses on individual constituents of the powerful chemical mixture provided by the Droso- 
phila male in his seminal fluid. Elucidation of the action of individual Acps permits a molecular understanding of reproductive interactions between females and males, including a model system for investigating the action of hormones or neuromodulators and an examination of the 'ecology' of fertilization in its normal setting. The study of Acps also can provide molecular probes with which to address evolutionary models for competition among individuals within a species.

Before the functions of individual seminal proteins can be identified, a comprehensive picture of the proteins secreted by male accessory glands is needed. Early studies involving differential cDNA hybridization screens to identify male-specific RNAs expressed in accessory glands (Schäfer, 1986; DiBenedetto et al, 1987; Monsma and Wolfner, 1988; Simmerl et al, 1995; Wolfner et al, 1997), or (in one case) identification of an accessory gland peptide by a functional assay (Chen et al, 1988), identified 18 Acp genes; according to Drosophila convention, these genes are named 'Acp' followed by the designation of their chromosome position. SDS-PAGE analysis of accessory gland proteins coupled with statistical tests based on the frequency of 'multiple hits' in the cDNA screens suggested that there were about 50-100 different Acps (Chen, 1991; Wolfner et al, 1997). Several characteristics of the 18 initial Acp genes (see Wolfner (1997) for review) were helpful in designing a comprehensive screen to identify all Acps. First, all 18 Acps have predicted signal sequences at their N-termini. Second, Acps expressed in the predominant cell type of the accessory gland (main cells, $96 \%$ of the secretory cells in the gland; Bertram et al, 1992) are not expressed in flies whose main cells are ablated with an intracellular toxin (Kalb et al, 1993). Finally, many Acp genes encode novel proteins or novel short peptides, and the sequences of several of these genes appear to be evolving rapidly apparently as a result of positive selection.

To identify the complete spectrum of Acp genes, a comprehensive EST screen was carried out by Swanson et al (2001) (Figure 1). ESTs made from male accessory gland RNA were selected initially for non-expression in females. The genes thus identified include a large number that encode novel proteins: $47 \%$ do not have relatives in non-Drosophila sequences in GenBank, suggesting that this group of ESTs is a rich source for new protein coding sequences. Swanson et al (2001) applied two additional criteria to focus on those ESTs most likely to encode Acps. First, genes that were not expressed when accessory gland main cells were ablated (Kalb et al, 1993) were considered Acps. Second, since Acps made in accessorygland cells other than main cells (Bertram et al, 1992) would be missed by this procedure, ESTs encoding proteins with predicted signal sequences were included in the group considered to be Acp genes. As with the 18 previously known Acp genes, the 57 genes selected by these criteria encode a range of molecules (Figure 2). They include predicted secreted small peptides or larger molecules that could be cleaved to yield multiple peptide hormones, predicted glycoproteins, and proteins with sequence motifs predictive of biochemical function. The latter class includes proteins predicted to be proteases, protease inhibitors or lipases. Based on multiple-hits in the EST screen, Swanson et al (2001) calculated that there are approximately $83 \mathrm{Acp}$ genes; this estimate is consist-

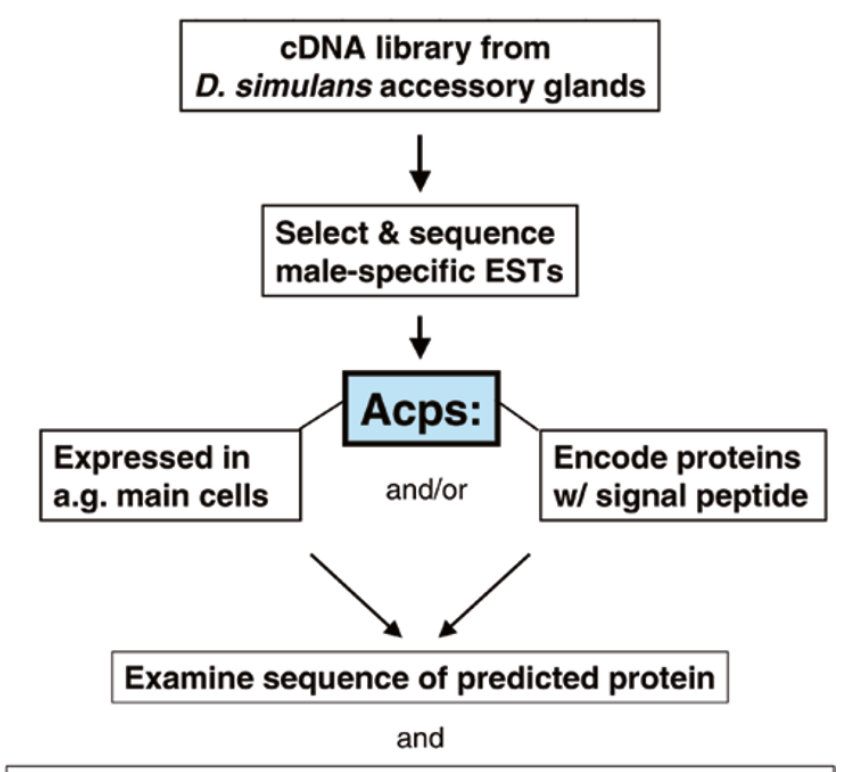

Estimate $d_{N} / d_{S}$ by comparing $D$. simulans Acp sequence to $D$. melanogaster genomic sequence

Figure 1 The 'evolutionary EST screen' that identified Acp genes, and identified candidate rapidly-evolving molecules. cDNA clones made from mRNA isolated from $D$. simulans accessory glands were screened for male-specific expression by hybridization to cDNA derived from $D$. simulans females. Clones that did not hybridize to female-derived cDNA were sequenced, and further screened for presence of a predicted signal sequence (von Heijne, 1983) and/or loss of expression in males lacking the primary cell type (main cells) of the accessory gland (Kalb et al, 1993). These putative Acps were examined for sequence features and, by comparison to the D. melanogaster genomic sequence (Adams et al, 2000), for signs of rapid evolutionary change. See Swanson et al (2001) for details.

\section{The 83 predicted Acps include:}

\section{- Peptides (25) or prohormone precursors} e.g. the ovulation hormone Acp26Aa (ovulin) the "sex peptide" Acp70A

\section{- Glycoproteins}

e.g. the sperm storage protein Acp36DE

\section{- Modifying enzymes \\ Proteases (9) \\ Protease inhibitors (8)}

e.g. the trypsin inhibitor Acp62F Lipases (6)

\section{- Many novel proteins $-\sim 11 \%$ with signs of rapid evolution}

Figure 2 Sequence classes of the 83 predicted Acps, isolated in several screens (Schäfer, 1986; DiBenedetto et al, 1987; Chen et al, 1988; Monsma and Wolfner, 1988; Simmerl et al, 1995; Wolfner et al, 1997; Swanson et al, 2001). The sequence and function of the four example Acps are described in: Acp26Aa (Monsma and Wolfner, 1988; Herndon and Wolfner, 1995; Heifetz et al, 2000; Chapman et al, 2001), Acp70A (Chen et al, 1988; Aigaki et al, 1991; Kubli, 1996; Moshitzky et al, 1996; Soller et al, 1997), Acp36DE (Wolfner et al, 1997; Neubaum and Wolfner, 1999; Chapman et al, 2000) and Acp62F (Wolfner et al, 1997; Lung et al, 2002). 
ent with those from the earlier studies. Ninety percent of these genes (75 predicted genes) are in hand.

\section{What are the functions of individual seminal fluid proteins?}

Now that predicted Acps have been identified, the function(s) of each Acp, and the way in which this function(s) is carried out, needs to be defined. Two types of genetic assay can be used to assign functions to individual Acps. The most conclusive is a knockout approach, in which an Acp is chosen for examination, often because of some interesting feature of its sequence or targeting. This Acp gene is knocked out, and the postmating response(s) that fails to occur in the absence of this particular Acp can be determined. This approach was successful for two Acps: the ovulation hormone Acp26Aa, which is discussed further below, and the sperm storage protein Acp36DE (Herndon and Wolfner, 1995; Neubaum and Wolfner, 1999; Chapman et al, 2000). Until recently, use of this method was limited owing to the difficulty in generating mutants in a gene defined only by sequence and without a phenotype that could be predicted in advance. A recently-reported technique for homologous recombination shows great potential for making it possible to generate future Acp knockouts more routinely (Rong and Golic, 2000, 2001). Given the difficulty of the knockout approach, other assays have also been used to identify potential functions of Acps. These assays usually scan several Acps at once for effects on a specific phenotype. For example, it can be determined whether ectopic expression of an Acp in unmated females causes them to display phenotypes that resemble any seen in mated female flies. This approach has led to the assignment of function to two Acps. Ectopic expression of the protease inhibitor Acp62F suggests a role in the survival cost of mating (Lung et al, 2002; discussed below). Ectopic expression and injection assays have also been used by the Kubli, Chen and Aigaki labs to show that Acp70A (sex peptide) can induce rejection behavior and increase egg-laying in females (Chen et al, 1988; Aigaki et al, 1991; Nakayama et al, 1997). Data from these labs and their collaborators are consistent with a model in which Acp70A stimulates the production of juvenile hormone BIII, which in turn stimulates oogenic progression (Moshitzky et al, 1996; Soller et al, 1997, 1999). Non-genetic assays also have helped to dissect Acp function. Immunofluorescence or GFP fusions have identified target tissues of Acps, which in turn provides insight into the mode of Acp action (Bertram et al, 1996; Lung and Wolfner, 1999; Heifetz et al, 2000; Ottiger et al, 2000). Direct biochemical or physiological assays, including some discussed below, have also provided functional information (Chen et al, 1988; Schmidt et al, 1989; Lung et al, 2001a; Lung et al, 2002), and correlations of allelic variation have in some cases suggested potential roles to consider for Acps (Clark et al, 1995).

The functions of four Acps are particularly well understood. Here, the focus is on two of them, the ovulation hormone Acp26Aa, and the protease inhibitor Acp62F (schematically shown in Figure 3a). The other two are the receptivity/egg-laying modulator Acp70A (Chen et al, 1988; Aigaki et al, 1991; Kubli, 1992, 1996; Moshitzky et al, 1996; Nakayama et al, 1997; Soller et al, 1997, 1999; Ottiger et al, 2000), and the sperm storage protein a

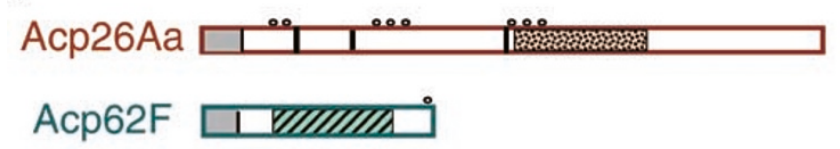

b

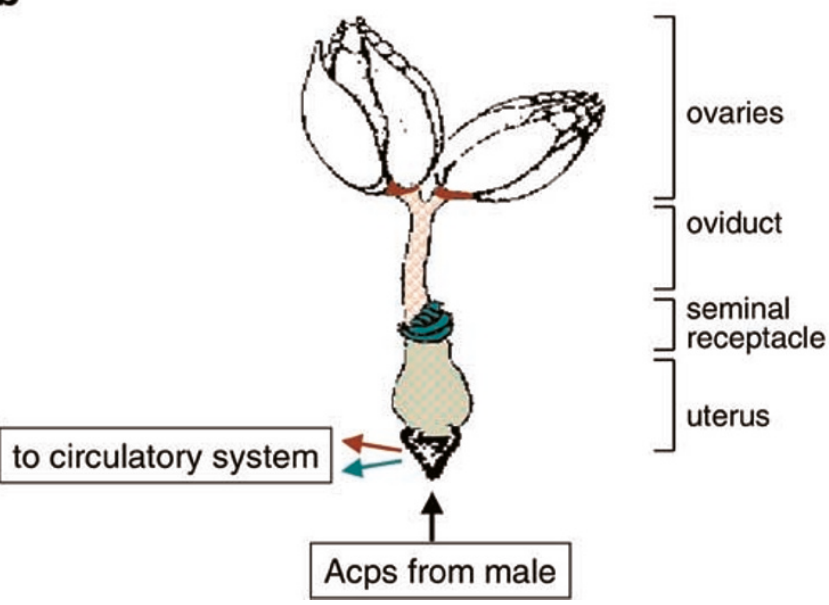

Figure 3 Features of Acp26Aa and Acp62F. (a) Schematic diagram of the primary sequence of Acp26Aa and Acp62F. Acp26Aa is a 264 amino acid predicted polypeptide (Monsma and Wolfner, 1988). It initiates with a predicted signal sequence (gray), and contains sites at which the protein is cleaved proteolytically once it is in the reproductive tract of a mated female fly (vertical bars) (Monsma and Wolfner, 1988; Monsma et al, 1990; Park and Wolfner, 1995). A region of Acp26Aa (brown, dotted: Monsma and Wolfner, 1988; Heifetz et al, 2000) has sequence similarity to califin C and ELH of Aplysia californica (Scheller et al, 1983; Rothman et al, 1986; Kurosky et al, 1998). Acp62F is a 115 amino acid predicted polypeptide (Wolfner et al, 1997), that initiates with a predicted signal sequence (gray) and has a region of sequence similarity to a class of secreted protease inhibitors from Ascaris (Peanasky et al, 1984a, b; Lung et al, 2002). (b) Schematic of the localization of Acp26Aa (brown) and Acp62F (green), shown on a simplified diagram of the mated female reproductive tract (spermathecae and female accessory glands have been omitted from the diagram, and 'oviduct' refers to common and lateral oviducts). Acp26Aa accumulates at the base of the ovaries, and is also seen in the lumen of the oviducts and uterus, possibly simply en route to the base of the ovaries (Heifetz et al, 2000). Acp $62 \mathrm{~F}$ is found in the seminal receptacle and in the uterine lumen (again presumably en route) (Lung et al, 2002). Some Acp26Aa and some Acp62F also cross the wall of the posterior vagina to enter the circulatory system of the female fly (Monsma et al, 1990; Lung and Wolfner, 1999).

Acp36DE (Bertram et al, 1996; Neubaum and Wolfner, 1999; Chapman et al, 2000).

The ovulation hormone Acp26Aa ('ovulin') Acp26Aa is a 264 amino acid-long polypeptide (Monsma and Wolfner (1988); Figure 3a). It is hydrophilic after its signal sequence and contains several pairs of basic amino acids (or single basic amino acids in cleavage contexts); thus, it resembles a precursor to multiple peptide hormones. Indeed when Acp26Aa enters the female fly, it undergoes proteolytic cleavage in a process that requires components donated by both male and female (Monsma et al, 1990; Park and Wolfner, 1995). The proteolytic products are consistent with ordered cleavage at the predicted cleavage sites, proceeding from the more 
$\mathrm{N}$-terminal to the more C-terminal sites. The amino acid sequence of Acp26Aa contains an interesting similarity to regions of a family of related egg-laying hormones made by the mollusk Aplysia californica (and also produced by cleavage from larger precursors; Scheller et al, 1983; Rothman et al, 1986).

A knockout mutation of Acp26Aa was without consequence to males themselves (Herndon and Wolfner, 1995). However, mates of males lacking Acp26Aa were affected in a single aspect of their post-mating physiology: they laid fewer eggs than normal on the first postmating day (Herndon and Wolfner, 1995), the time of action of Acps (Kalb et al, 1993). Therefore Acp26Aa was necessary to stimulate the egg-laying process in mated females. Females mated to males that lacked the protein still show some egg-laying elevation, indicating that molecules besides Acp26Aa also stimulate egg-laying. One of these molecules is likely to be Acp $70 A$, based on the results cited above (Chen et al, 1988; Aigaki et al, 1991). To gain a full understanding of Acp26Aa's action, it was necessary to understand how it stimulated the egg-laying process, and whether its action was redundant with, or complementary to, that of other Acps such as Acp70A.

The egg-laying process involves multiple steps (Soller et al, 1999; Heifetz et al, 2000). Eggs have to be produced (via oogenesis), pass a checkpoint during oogenesis, be released from ovaries (ovulated), move down the oviducts to the uterus, be released from the uterus, and be laid. In principle an Acp could act at any or all stages in the process. To determine at which step(s) Acp26Aa exerts its effect, we developed an assay to measure the progress through each step individually, by directly quantifying the number of eggs at each stage of their movement though the female reproductive tract (Heifetz et al, 2000). Then the progress through each stage of the egg-laying process was compared in females who had received Acp26Aa from their mates relative to females who had mated to males lacking Acp26Aa. It was discovered that just one step, the initial release of oocytes at ovulation, is dependent on the transfer of Acp26Aa; because of this function, Acp26Aa has been renamed 'ovulin' (Heifetz et al, 2000). Our data indicate that Acp26Aa stimulates the immediate release of eggs following mating - its effects are evident by $1.5 \mathrm{~h}$ post-mating and persist until about $6 \mathrm{~h}$ post-mating. This early time of action leads to the model that Acp26Aa causes the release of mature oocytes accumulated in the ovary before the mating. Since such action affects only eggs that are already in existence, it would not be expected to exact a further energetic cost on the female. Consistent with this expectation, Chapman et al (2001) showed that Acp26Aa does not impact the lifespan of mated females. Acp26Aa releases eggs while sperm are still being stored. Those eggs would therefore be expected to be fertilized less efficiently than eggs ovulated later, when sperm are fully stored and in position to be released efficiently; this too was observed (Chapman et al, 2001). Thus, the function of Acp26Aa appears to be to stimulate ovulation shortly after mating, to 'clear' mature eggs from the ovary. This sacrifice of a few mature eggs would relieve the pre-mating arrest of oogenesis caused by the accumulation of mature, unovulated eggs. It could potentially also allow subsequent synchronization of egg and sperm release to permit optimal rates of fertilization.

How does Acp26Aa cause this oocyte release?
Acp26Aa might act directly on targets in the reproductive tract, since it has been seen to bind to sites at the base of the ovaries (Heifetz et al (2000); summarized in Figure 3b). Alternatively, since Acp26Aa also enters the circulatory system of the female fly (Monsma et al, 1990; Lung and Wolfner, 1999), it could trigger endocrine or neural signals that stimulate ovulation. In either case, Acp26Aa action must ultimately affect reproductive tract tissues at or near the base of the ovary. To identify the molecular consequence of Acp26Aa action in the Drosophila female reproductive tract, we are presently examining these tissues for the role of Acps, and Acp26Aa in particular, in triggering vesicle exocytosis and/or alteration in levels of neuromodulators that cause muscle contraction and thus might be involved in inducing ovulation (Trent et al, 1983; Lange et al, 1986, 1991; Bamji and Orchard, 1995; Lange and Nykamp, 1996; Monastirioti et al, 1996; Yamauchi et al, 1997; Waggoner et al, 1998).

In summary, Acp26Aa (ovulin) has been identified as a mediator of one specific step in the egg-laying process: the release of mature oocytes from the ovary. Studies in the Kubli lab indicate that the stimulation of egg-laying by another Acp, Acp70A (sex peptide), occurs by stimulating oogenesis (Soller et al, 1997, 1999). Thus, although at least two Acps modulate the egg-laying process, they act on different steps. This suggests that seminal fluid is a potent chemical cocktail, whose components independently regulate individual steps in multi-step processes. Having independent regulators allows each stage of the process to be tuned to maximal efficiency on its own, as well as in concert with other steps. Moreover, given the fact that many Acps appear to be evolving rapidly (see below), it may be best to have critical processes controlled at several steps, independently. This could allow essential reproductive processes to accommodate changes in individual Acps better than if the entire pathway were controlled by one Acp, or by a group of Acps acting together in a single biochemical complex, on only one physiological step.

\section{The protease inhibitor Acp62F}

Action of Acps contributes to the survival cost of mating. In other words, not all gifts are pleasant or benign. How could an Acp, deposited into the reproductive tract of a female (and hence, topologically on her outside) cause a systemic detrimental effect on the female? It turns out that most Acps, including Acp62F (and Acp26Aa as noted above), can cross a permeable region at the posterior of the female reproductive tract to enter the circulatory system of the female and gain access to all her other tissues, including neural and endocrine tissues (Lung and Wolfner, 1999). The basis for this entry is not apparent in Acps' sequences, and may be simply due to non-selective transport of Acps that are below a given size cutoff or that are not bound to sperm or to the reproductive tract wall. Entry of Acps into the female's circulation gives them the means to exert systemic effects on her physiology and behavior.

To identify an Acp that might be an agent for decreasing the lifespan of the mated female, we tested eight separate Acps for effects on viability (Lung et al, 2002). We introduced each Acp individually into D. melanogaster and compared the effects of ectopic expression of each Acp on viability, relative to expression of a control protein. Only one Acp, Acp62F, was toxic to preadult 
Drosophila and, upon multiple rounds of expression, to adults.

What is the biochemical action of Acp62F, and can this explain its toxicity? The sequence of Acp62F has several intriguing similarities, but the most extensive is to a class of extracellular protease inhibitors produced by Ascaris worms (Peanasky et al, 1984a, b; schematically shown in Figure 3a). Moreover, the similarity extends to the predicted 3D structure of the proteins: the structure of the Ascaris inhibitors has been solved, and Acp62F's sequence threads along it very well. This threading allows the prediction of the active site of Acp62F; it has the characteristics of a trypsin inhibitory site. In vitro assays showed that Acp62F indeed inhibits trypsin (and not other proteases like elastase) and that mutations of its predicted active site either abolish this biochemical activity or change its specificity exactly as predicted from effects of the mutations on the protein's 3D structure. Thus Acp62F is a trypsin inhibitor in Drosophila seminal fluid that is transferred to females during mating (Lung et al, 2002).

Why might a seminal protease inhibitor be toxic? About $10 \%$ of the Acp62F transferred to a female enters her circulatory system, through the permeable region mentioned above (Lung and Wolfner (1999); summarized in Figure 3b). Thus, Acp62F could act on a cell type or process anywhere in the female. One possible model is that entry of this proteolysis regulator into the circulatory system places it at a site from which it can alter the progress of essential proteolytic cascades. These cascades include those that are needed for the fly's immunity to microbial parasites and could thus make females more susceptible to infection (eg see Imler and Hoffmann (2000) for review), thereby shortening their life. Altering the efficiency of other metabolic or endocrine proteolytic cascades could also, in theory, affect pathways essential for robust viability. Small amounts of Acp62F, introduced repeatedly upon multiple matings could therefore result in a decrease in female viability - part of the measured survival cost of mating. Further experiments are required to determine the nature of and reason for the toxicity of Acp62F, and whether it truly is an agent of the survival cost of mating. In light of this hypothesis, it is interesting to note that seven other Acps are predicted to be protease inhibitors. Their specificities may differ from that of Acp62F, and thus they could affect different proteolytic cascades. If any of them (or any of the nine predicted proteases) also enters the circulation of the mated female, they too could alter the balance of precisely-tuned proteolytic cascades. Thus, if effects on proteolytic cascades contribute to the survival cost of mating, there is the potential that this cost could reflect the aggregate effect of several Acps.

But if protease inhibitors like Acp62F decrease the lifespan of the mated female fly, why would their presence in seminal fluid (and entry into the circulatory system) be tolerated through evolution? An attractive explanation is that these seminal protease inhibitors play a positive role that is so valuable to reproductive success that it would be disadvantageous to lose these proteins. Indeed, in mammals, protease inhibitors have been suggested to play a positive role in male fertility. For example, knockout of the mouse seminal protease inhibitor protease nexin-1 (PN-1) results in male infertility (Murer et al, 2001), and altered levels or function of PN-1 or of the serpin protein $C$ inhibitor are observed in seminal fluid of some infertile men (He et al, 1999; Murer et al, 2001). The short-term benefit of a protease inhibitor in Drosophila seminal fluid could outweigh long-term negative consequences, particularly if those consequences occur after many progeny have been produced. In addition, evolution could generate situations in which the negative effects of Acp62F were decreased by having proteins in circulation that sequester it or dampen its activity, or by keeping the amount transferred to a level sufficient for its positive function but below the threshold for serious or immediate negative effects. Indeed the toxicity seen in our experiments occurred at high levels of Acp62F, and multiple matings are required to detect the survival cost of mating under normal conditions. What sorts of positive roles could seminal protease inhibitors play? First, a protease inhibitory Acp may be necessary to regulate the proteolysis of other proteins, such as Acp26Aa (Monsma et al, 1990; Park and Wolfner, 1995). Such action might protect Acps from non-specific proteolysis in the accessory gland, keeping them intact and at high levels, or it could regulate the rate or position of Acp processing in the mated female's reproductive tract. Second, protease inhibitors might be needed to regulate the coagulation of seminal fluid once it is in the female, as has been proposed for the mammalian protein $\mathrm{C}$ inhibitor (Kise et al (1996); see Robert and Gagnon (1999) for review). Third, a protease inhibitor may be needed to protect sperm from proteolytic degradation of their surface proteins and to keep them stable in storage. In cattle, the seminal plasma inhibitor BUSI-II has been proposed to protect sperm from premature acrosome reaction (Veselsky and Cechova, 1980). Intriguingly suggestive of a spermprotective role for Acp62F, this protein is present in $D$. melanogaster sperm storage organs along with sperm (Lung et al, 2002; summarized in Figure 3b). Reproductive functions such as these could require inhibition of many, or many types, of proteases; perhaps this accounts for the number and variety of protease inhibitors in seminal fluid.

In summary, D. melanogaster seminal fluid contains at least one active regulator of proteolysis, the protease inhibitor Acp62F. The predicted sequences of 16 other Acps suggest they too could modulate proteolysis (Wolfner et al, 1997; Swanson et al, 2001), though this still requires biochemical verification. Protease inhibitors in seminal fluid could play positive roles in regulating seminal protein proteolysis and/or in contributing to the functional viability of sperm. Yet, the entry of these proteins into the female's circulation, as occurs for Acp62F, could potentially interfere with the proper efficiency of essential proteolytic cascades. This could cause negative consequences to the female, including toxicity, as is seen upon ectopic expression of Acp62F.

\section{Acps give investigators tools with which to address evolutionary questions}

Action of Acps requires interaction at the molecular level between female and male. The reproductive interests that impact on the evolution of Acps, and their receptors and response pathways can be common, such as increased progeny production, but can also be at odds between the sexes. For example, while it may be advantageous to a male that his mate avoids re-mating so that his stored 
sperm are not subject to competition, it may be advantageous to the female to have multiple partners, who can provide different genetic and chemical 'donations'. Several excellent hypotheses for the evolution of male or female sexual traits have been advanced (for example: Eberhard, 1996; Rice, 1996; Holland and Rice, 1999; Arnqvist et al, 2000; Birkhead, 2000; Gavrilets, 2000; Chippindale et al, 2001; Gavrilets et al, 2001; Hosken et al, 2001). One proposes that there is an evolutionary crosstalk between the sexes that could drive rapid evolution of reproductive molecules: male molecules could 'manipulate' a female's physiology and females could evolve to become inured to this effect. This, in turn, could drive rapid evolution of the male molecules.

Among the first 18 Acps, the sequences of several, including Acp26Aa, show signs of rapid evolution consistent with positive Darwinian selection (Aguadé et al, 1992; Tsaur and Wu, 1997; Aguadé, 1998; Tsaur et al, 1998, 2001; Begun et al, 2000). The EST screen described above (Swanson et al, 2001) not only provided raw material to test systematically and in a large scale whether an unusually high fraction of Acps show signs consistent with rapid evolution, but in fact was designed to pinpoint Acps that appear to be the target of positive selection. The screen was not, in other words, an ordinary EST screen; it was an 'evolutionary EST screen'. The ESTs were made from accessory gland RNA from Drosophila simulans, a close relative of $D$. melanogaster. The genomes of these flies show an average of $11 \%$ base pair differences (Begun and Whitley, 2000).

Sequences of the ESTs were compared with the D. melanogaster genomic sequence (Adams et al, 2000). This allowed immediate identification of Acps whose sequences had characteristics suggesting that they might be evolving rapidly; these were Acps whose $\mathrm{dN} / \mathrm{dS}$ ratios (number of nonsynonymous substitutions/ nonsynonymous sites relative to synonymous substitutions/ synonymous sites) were greater than 1 (the ratio for sequences changing at the neutral rate) (Hughes and Nei, 1988). Eleven percent of Acps identified in the EST screen show elevated $\mathrm{dN} / \mathrm{d} S$ ratios, suggesting that they might be candidates for genes showing rapid evolution (Swanson et al, 2001). This frequency is much higher than that observed for ESTs from other tissues and is consistent with the idea that some Acps might be involved in molecular interchanges between the sexes according to models of sexually antagonistic coevolution (Rice, 1996). This new method can be used to identify rapidly-evolving genes in any pair of related species of which one has a fully characterized genomic sequence.

In conclusion, the male Drosophila gives his mate, during mating, a potent mix of seminal proteins (and sperm) that can alter the physiology, behavior and lifespan of the female. Individual Acp molecules influence the rate or occurrence of particular steps within multi-step processes and act together to regulate these processes to full efficiency. Gene knockout approaches, and ectopic expression assays have begun to identify Acps that mediate increased ovulation, decreased receptivity or storage of sperm as well as a candidate for the Acp that decreases the lifespan of the mated female fly. Future studies will identify the molecular mechanisms, receptors and pathways through which these molecules act. Acps are interesting not only for their physiological and hormonal roles, but also for their evolutionary dynamics. An unusually high fraction of these proteins shows signs consistent with having been subjected to positive selection. The identification of Acp genes gives molecular tools for investigation of the evolutionary dynamics of reproductive proteins.

\section{Acknowledgements}

I thank the present and former members of my laboratory, and our collaborators C Aquadro, T Chapman, A Clark, L Partridge and members of the 'Seminal Peptides Group', for many enjoyable discussions about Acps, and the NSF and NIH for funding. I also thank the organizers of the Genetics Society meeting on 'Sex' (March, 2001) for inviting me to give the talk that formed the basis for this article. S Albright, C Aquadro, M Bloch Qazi, T Chapman, Y Heifetz, L McGraw and J Mueller provided many helpful comments on the manuscript. I thank C Aquadro for the gift of this article's title. I apologize to those whose work could not be cited, or is cited only via a review article, owing to the journal's limitations on citations.

\section{References}

Adams MD, Celniker SE, Holt RA, Evans CA, Gocayne JD, Amanatides PG et al (2000). The genome sequence of Drosophila melanogaster. Science 287; 2185-2195.

Aguadé M (1998). Different forces drive the evolution of the Acp26Aa and Acp26Ab accessory gland genes in the Drosophila melanogaster species complex. Genetics 150: 1079-1089.

Aguadé M, Miyashita N, Langley C (1992). Polymorphism and divergence in the Mst26A male accessory gland gene region in Drosophila. Genetics 132: 755-770.

Aigaki T, Fleischmann I, Chen PS, Kubli E (1991). Ectopic expression of sex peptide alters reproductive behavior of female D. melanogaster. Neuron 7: 557-563.

Alcock J (1998). Animal Behavior: An Evolutionary Approach. Sinauer Associates: Sunderland, Mass.

Antony, C, Jallon J (1982). The chemical basis of sex recognition in Drosophila melanogaster. J Ins Physiol 28: 873-880.

Arnqvist G, Edvardsson M, Friberg U, Nilsson T (2000). Sexual conflict promotes speciation in insects. Proc Natl Acad Sci USA 97: 10460-10464.

Bairati A (1968). Structure and ultrastructure of the male reproductive system of Drosophila melanogaster Meig. 2. The genital duct and accessory glands. Monit Zool Ital 2: 105-182.

Bamji SX, Orchard I (1995). Pharmacological profile of octopamine and 5HT receptor on the lateral oviducts of the cockroach, Periplaneta americana. Arch Ins Bioch Phys 28: 49-62.

Begun DJ, Whitley P (2000). Reduced X-linked nucleotide polymorphism in Drosohila simulans. Proc Natl Acad Sci USA 97: 5960-5965.

Begun DJ, Whitley P, Todd BL, Waldrip-Dail HM, Clark AG (2000). Molecular poplation genetics of male accessory gland proteins in Drosophila. Genetics 156: 1879-1888.

Bertram M, Akerkar GA, Ard RL, Gonzalez C, Wolfner MF (1992). Cell type specific gene expression in the Drosophila melanogaster male accessory gland. Mechs Dev 38: 33-40.

Bertram MJ, Neubaum DM, Wolfner MF (1996). Localization of the Drosophila accessory gland protein Acp36DE in the mated female suggests a role in sperm storage. Insect Bioch Mol Biol 26: 971-980.

Birkhead T (2000). Promiscuity: an evolutionary history of sperm competition. Harvard University Press: Boston MA.

Boswell RE, Mahowald AP (1985). tudor, a gene required for assembly of the germ plasm in Drosophila melanogaster. Cell 43: 97-104.

Bridges CB (1916). Non-disjunction as proof of the chromosome theory of heredity. Genetics 1: 1-52. 
Cavener DR, MacIntyre RJ (1983). Biphasic expression and function of glucose dehydrogenase in Drosophila melanogaster. Proc Natl Acad Sci USA 80: 6286-6288.

Chapman T, Neubaum DM, Wolfner MF, Partridge L (2000). The role of male accessory gland protein Acp36DE in sperm competition in Drosophila melanogaster. Proc Roy Soc Lond B Biol Sci 267: 1097-1105.

Chapman T, Herndon LA, Heifetz Y, Partridge L, Wolfner MF (2001). The Acp26Aa seminal fluid protein is a modulator of early egg-hatchability in Drosophila melanogaster. Proc Roy Soc Lond B Biol Sci 268: 1647-1654.

Chapman T, Liddle LF, Kalb JM, Wolfner MF, Partridge L (1995). Cost of mating in Drosophila melanogaster females is mediated by male accessory gland products. Nature 373: 241-244.

Chen PS (1991). Biochemistry and molecular regulation of the male accessory gland secretions in Drosophila (Diptera). Annals Soc ent Fr 27: 231-244.

Chen PS (1996). The accessory gland proteins in male Drosophila: structural, reproductive and evolutionary aspects. Experientia 52: 503-510.

Chen PS, Stumm-Zollinger E, Aigaki T, Balmer J, Bienz M, Bohlen P (1988). A male accessory gland peptide that regulates reproductive behavior of female D. melanogaster. Cell 54: 291-298.

Chippindale AK, Gibson JR, Rice WR (2001). Negative genetic correlation for adult fitness between sexes reveals ontogenetic conflict in Drosophila. Proc Natl Acad Sci USA 98: 1671-1675.

Clark AG, Aguadé M, Prout T, Harshman LG, Langley CH (1995). Variation in sperm displacement and its association with accessory gland protein loci in Drosophila melanogaster. Genetics 139: 189-201.

Clark AG, Begun DJ (1998). Female genotypes affect sperm displacement in Drosophila. Genetics 149: 1487-1493.

Clark AG, Begun DJ, Prout T (1999). Female x male interactions in Drosophila sperm competition. Science 283: 217-220.

DiBenedetto AJ, Lakich DM, Kruger WD, Belote JM, Baker BS, Wolfner MF (1987). Sequences expressed sex-specifically in Drosophila melanogaster adults. Dev Biol 119: 242-251.

Eberhard WG (1996). Female Control: Sexual Selection by Cryptic Female Choice. Princeton Univ. Press: Princeton, NJ.

Fowler K, Partridge L (1989). A cost of mating in female fruitflies. Nature 338: 760-761.

Gavrilets S (2000). Rapid evolution of reproductive barriers driven by sexual conflict. Nature 403: 886-889

Gavrilets S, Arnqvist G, Friberg U (2001). The evolution of female mate choice by sexual conflict. Proc R Soc Lond B Biol Sci 268: 531-539.

Gilbert DG (1981). Ejaculate esterase and initial sperm use by female Drosophila melanogaster. J Insect Physiol 27: 641-650.

Gilbert DG, Richmond RC, Sheehan KB (1981). Studies of esterase 6 in Drosophila melanogaster. V. Progeny production and sperm use in females inseminated by males having active or null alelles. Evolution 35: 21-37.

Greenspan RJ, Ferveur JF (2000). Courtship in Drosophila. Annu Rev Genet 34: 205-232.

Hall JC (1994). The mating of a fly. Science 264: 1702-1714.

Harshman LG, Prout T (1994). Sperm displacement without sperm transfer in Drosophila melanogaster. Evolution 48: 758766.

He S, Lin YL, Liu YX (1999). Functionally inactive protein C inhibitor in seminal plasma may be associated with infertility. Mol Hum Reprod 5: 513-519.

Heifetz Y, Lung O, Frongillo EA, Wolfner MF (2000). The Drosophila seminal fluid protein Acp26Aa stimulates release of oocytes by the ovary. Curr Biol 10: 99-102.

Heifetz Y, Tram U, Wolfner MF (2001). Male contributions to egg production: the role of accessory gland products and sperm in Drosophila melanogaster. Proc $R$ Soc Lond B Biol Sci 268: 175-180.

Herndon LA, Wolfner MF (1995). A Drosophila seminal fluid protein, Acp26Aa, stimulates egg-laying in females for one day following mating. Proc Natl Acad Sci USA 92: 1011410118.

Hihara F (1981). Effects of the male accessory gland secretion on oviposition and remating in females of Drosophila melanogaster. Zool Mag 90: 303-316.

Holland B, Rice WR (1999). Experimental removal of sexual selection reverses intersexual antagonistic coevolution and removes a reproductive load. Proc Natl Acad Sci USA 96: 5083-5088.

Hosken DJ, Garner TW, Ward PI (2001). Sexual conflict selects for male and female reproductive characters. Curr Biol 11:: 489-493.

Hughes AL, Nei M (1988). Pattern of nucleotide substitution at major histocompatibility complex class I loci reveals overdominant selection. Nature 335: 167-170.

Imler JL, Hoffmann JA (2000). Signaling mechanisms in the antimicrobial host defense of Drosophila. Curr Opin Microbiol 3: $16-22$.

Jallon J (1984). A few chemical words exchanged by Drosophila during courtship and mating. Behav Genet 14: 441-478.

Kalb J, DiBenedetto AJ, Wolfner MF (1993). Probing the function of Drosophila melanogaster accessory glands by directed cell ablation. Proc Natl Acad Sci USA 90: 8093-8097.

Kise H, Nishioka J, Kawamura J, Suzuki K (1996). Characterization of semenogelin II and its molecular interaction with prostate-specific antigen and protein C inhibitor. Eur J Biochem 238: 88-96.

Krebs JR, Davies NB (1993). An Introduction to Behavioral Ecology. Blackwell Scientific Publications: Oxford, UK.

Kubli E (1992). My favorite molecule: the sex peptide. Bioessays 14: 779-784.

Kubli E (1996). The Drosophila sex-peptide: a peptide pheromone involved in reproduction. Adv Devel Biochem 4: 99-128.

Kurosky A, Gorham EL, van Heumen WRA, Garcia AT, Smith JS, Mifflin RC (1998). Expression and genetic variation of the Aplysia egg-laying hormone gene family in the atrial gland. Invertebrate Neurosci 2: 261-271.

Lange AB, Nykamp DA (1996). Signal transduction pathways regulating the contraction of an insect visceral muscle. Arch Insect Biochem Physiol 33: 183-196.

Lange AB, Orchard I, Adams ME (1986). Peptidergic innervation of insect reproductive tissue: the association of proctolin with oviduct viscercal musculature. J Comp Neurol 254: 279-286.

Lange AB, Orchard I, Brugge VA (1991). Evidence for the involvement of a SchistoFLRFamide-like peptide in the neural control of locust oviduct. Comp Biochem Physiol A 168: 383-391.

Ludwig MZ, Uspensky II, Ivanov AI, Kopantseva MR, Dianov CM, Tamarina NA et al (1991). Genetic control and expression of the major ejaculatory bulb protein PEB-me in Drosophila melanogaster. Bioch Genet 29: 215-240.

Lung O, Kuo L, Wolfner MF (2001a). Drosophila males transfer antibacterial proteins from their accessory gland and ejaculatory duct to their mates. J Insect Physiol 47: 617-622.

Lung O, Kuo L, Wolfner MF (2001b). Identification of and characterization of the major $D$. melanogaster mating plug protein. Insect Bioch Mol Bio 31: 543-551.

Lung O, Tram U, Finnerty C, Eipper-Mains M, Kalb JM, Wolfner MF (2002). The Drosophila melanogaster seminal fluid protein Acp62F is a protease inhibitor that is toxic upon ectopic expression. Genetics (in press).

Lung O, Wolfner MF (1999). Drosophila seminal fluid proteins enter the circulatory system through the walls of the posterior vagina. Insect Biochem Mol Biol 29: 1043-1052.

Manning A (1962). A sperm factor affecting the receptivity of Drosophila melanogaster females. Nature 194: 252-253.

Manning A (1967). The control of sexual receptivity in female Drosophila. Anim Behav 15: 239-250.

Markow TA, Coppola A, Watts TD (2001). How Drosophila males make eggs: it is elemental. Proc $R$ Soc Lond B Biol Sci 268: 1527-1532.

Monastirioti M, Linn CE, White K (1996). Characterization of 
Drosophila tyramine beta-hydroxylase gene and isolation of mutant flies lacking octopamine. J Neurosci 16: 3900-3911.

Monsma SA, Harada HA, Wolfner MF (1990). Synthesis of two Drosophila male accessory gland proteins and their fate after transfer to the female during mating. Dev Biol 142: 465-475.

Monsma SA, Wolfner MF (1988). Structure and expression of a Drosophila male accessory gland gene whose product resembles a peptide pheromone precursor. Genes Dev 2: 1063-1073.

Moshitzky P, Fleischmann I, Chaimov N, Saudan P, Klauser S, Kubli E et al (1996). Sex-peptide activates juvenile hormone biosynthesis in the Drosophila melanogaster corpus allatum. Arch Insect Bioch Physiol 32: 363-374.

Murer V, Spetz JF, Hengst U, Altrogge LM, deAgostini A, Monard D (2001). Male fertility defects in mice lacking the serine protease inhibitor protease nexin-1. Proc Natl Acad Sci USA 98: 3029-3033.

Nakayama S, Kaiser K, Aigaki T (1997). Ectopic expression of sex peptide in a variety of tissues in Drosophila females using the P[GAL4] enhancer-trap system. Mol Gen Genet 254: 449455.

Neubaum DM, Wolfner MF (1999). Drosophila females require a seminal fluid protein, Acp36DE, to store sperm efficiently. Genetics 153: 845-857.

Ottiger M, Soller M, Stocker RF, Kubli E (2000). Binding sites of Drosophila melanogaster sex peptide pheromones. I Neurobiol 44: 57-71.

Park M, Wolfner MF (1995). Male and female cooperate in the processing of a Drosophila melanogaster seminal fluid protein. Dev Biol 171: 694-702.

Parker GA (1970). Sperm competition and its evolutionary consequences in the insects. Biol Revs 45: 525-567.

Peanasky RJ, Bentz Y, Homandberg GA, Minor ST, Babin DR (1984). The isoinhibitors of chymotrypsin/elastase from Ascaris lumbricoides: the reactive site. Arch Biochem Biophys 232 135-142.

Peanasky RJ, Bentz Y, Paulson B, Graham DL, Babin DR (1984). The isoinhibitors of chymotrypsin/elastase from Ascaris lumbricoides: isolation by affinity chromatography and association with the enzymes. Arch Biochem Biophys 232: 127-134.

Rice WR (1996). Sexually antagonistic male adaptation triggered by experimental arrest of female evolution. Nature 381: 232234.

Robert M, Gagnon C (1999). Semenogelin I: a coagulum forming, multifunctional seminal vesicle protein. Cell Mol Life Sci 55: 944-960.

Rong YS, Golic KG (2000). Gene targeting by homologous recombination in Drosophila. Science 288: 2013-2018.

Rong YS, Golic KG (2001). A targeted gene knockout in Drosophila. Genetics 157: 1307-1312.

Rothman BS, Hawke DH, Brown RO, Lee TD, Dehghan AA, Shively JE, Mayeri E (1986). Isolation and primary structure of the califins, three biologically active egg-laying hormonelike peptides from the atrial gland of Aplysia californica. J Biol Chem 261: 1616-1623.

Schäfer U (1986). Genes for male-specific transcripts in Drosophila melanogaster. Mol Gen Genet 202: 219-225.

Scheller RH, Jackson JF, McAlister LB, Rothman BS, Mayeri E, Axel R (1983). A single gene encodes multiple neuropeptides mediating a stereotyped behavior. Cell 32: 7-22.

Schmidt T, Stumm-Zollinger E, Chen PS (1989). A male accessory gland peptide with protease inhibitory activity in Drosophila funebris. I Biol Chem 264: 9745-9749.

Simmerl E, Schäfer M, Schäfer U (1995). Structure and regulation of a gene cluster for male accessory gland transcripts in Drosophila melanogaster. Insect Biochem Molec Biol 25: 127-137.

Soller M, Bownes M, Kubli E (1997). Mating and sex peptide stimulate the accumulation of yolk in oocytes of Drosophila melanogaster. Eur J Biochem 243: 732-738.

Soller M, Bownes M, Kubli E (1999). Control of oocyte maturation in sexually mature Drosophila females. Dev Biol 208: 337-351.

Swanson WJ, Clark AG, Waldrip-Dail HM, Wolfner MF, Aquadro CF (2001). Evolutionary EST analysis identifies rapidly evolving male reproductive proteins in Drosophila. Proc Natl Acad Sci USA 98: 7375-7379.

Tram U, Wolfner MF (1998). Seminal fluid regulation of female sexual attractiveness in Drosophila melanogaster. Proc Natl Acad Sci USA 95: 4051-4054.

Tram U, Wolfner MF (1999). Male seminal fluid proteins are essential for sperm storage in Drosophila melanogaster. Genetics 153: 837-844.

Trent C, Tsung N, Horvitz R (1983). Egg-laying defective mutants of the nematode Caenorhabditis elegans. Genetics 104: 619-647.

Tsaur SC, Ting CT, Wu C-I (1998). Positive selection driving the evolution of a gene of male reproduction, Acp26Aa, of Drosophila: II. Divergence versus polymorphism. Mol Biol Evol 15: 1040-1046.

Tsaur SC, Ting CT, Wu CI (2001). Sex in Drosophila mauritiana: a very high level of amino acid polymorphism in a male reproductive protein gene, Acp26Aa. Mol Biol Evol 18: 22-26.

Tsaur SC, Wu C-I (1997). Positive selection and the molecular evolution of a gene of male reproduction, Acp26Aa of Drosophila. Mol Biol Evol 14: 544-549.

Veselsky L, Cechova D (1980). Distribution of acrosin inhibitors in bull reproductive tissues and spermatozoa. Hoppe Seylers $Z$ Physiol Chem 361: 715-722.

von Heijne G (1983). Patterns of amino acids near signal sequence cleavage sites. Eur J Biochem 135: 17-21.

Waggoner LE, Zhou GT, Schafer RW, Schafer WR (1998). Control of alternative behavioral states by serotonin in Caenorhabditis elegans. Neuron 21: 203-214.

Wolfner MF (1997). Tokens of love: functions and regulation of Drosophila male accessory gland products. Insect Bioch $\mathrm{Mol}$ Biol 27: 179-192.

Wolfner MF, Harada HA, Bertram MJ, Stelick TJ, Kraus KW, Kalb JM et al (1997). New genes for accessory gland proteins in D. melanogaster. Insect Biochem Mol Biol 27: 825-834.

Xue L, Noll M (2000). Drosophila female sexual behavior induced by sterile males showing copulation complementation. Proc Natl Acad Sci USA 97: 3272-3275.

Yamauchi J, Miyazaki T, Iwasaki S, Kishi I, Kuroshima M, Tei $C$ et al (1997). Effects of nitric oxide on ovulation and ovarian steroidogenesis and prostaglandin production in the rabbit. Endocrinology 138: 3630-3637.

Zarkower D (2001). Establishing sexual dimorphism: conservation amidst diversity? Nat Rev Genet 2: 175-185. 